\title{
Industrial Automation and Process Control System Using Can Bus
}

\author{
B. Meena Kumari ${ }^{1}$, M. Satya Sailaja ${ }^{2}$
}

\begin{abstract}
The main aim of this project is to provide more safety to industry machines by avoiding operating from over temperatures and also provide constant lighting by controlling artificial lights depends upon the ambient light intensity and magnetic reflections.
\end{abstract}

Keywords: ARM7, CAN, LM35, LDR, Reed-Switch.

\section{Introduction}

Industrial automation and process control greatly reduces the need for human sensory and mental requirements as well. Most complex industrial automation processes and systems can be automated. A major advantage of industrial automation and process control is the increased emphasis on flexibility and convertibility in the manufacturing process.

\section{Proposed System}

As our project is proposed that in industry there is lot of reflections to be avoided to that we connecting sensor devices to arm for detecting the errors and automatically correct the error without manual work.

\section{System Architecture}

It is composed of two parts

Transmitter side: This side is consists of three types of sensors such as temperature sensor, LDR sensor, reed switch. These sensors are used to measure the signals from the surrounding of machinery. After measurement these analog signals are converted into digital signals and compared with the actual signals. If any discrepancy occurs between the measured and actual signals, then it is considered as emergency. The ARM LPC 2148 processor plays an important role in controlling all the devices. It has an inbuilt $\mathrm{A} / \mathrm{D}$ converter. The ARM7TDMI core is the industry's most widely used 32-bit embedded RISC microprocessor solution. Optimized for cost and power sensitive applications, the ARM7TDM solution provides the low power consumption, small size, and high performance needed in portable, embedded applications. CAN transmitter and receivers connected to ARM these will collect the signals and transfer to the receiver side.

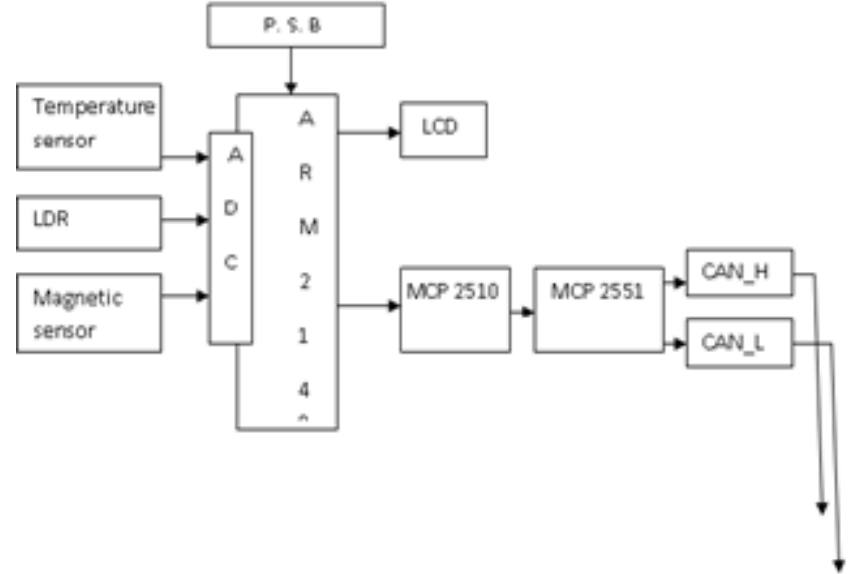

Figure 1: Block Diagram at Transmitter Side.

Receiver Side: This side consists CAN transmitter and receiver that are used to transfer the error to arm to detect the errors and data is transfer to ARM. Those values are displayed on the LCD and those values are compared with the actual values and set correct value.

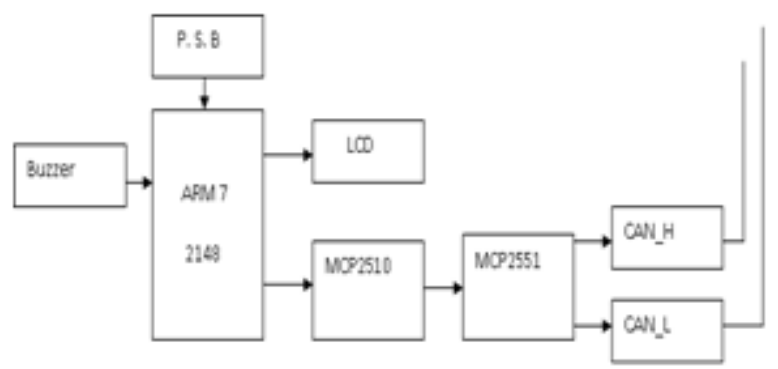

Figure 2: Block Diagram at Receiver Side

\section{Design Description}

All the design of proposed system is described in the following.

\section{Hardware Description}

In our project ARM controller is used. To measure temperature there will be temperature sensors to convert the output of sensor into electrical form. LDR for light intensity and Reed switch for magnetic reflections.

\section{i) ARM 7-LPC2148}

The ARM7TDMI-S processor also employs a unique architectural strategy known as Thumb, which makes it 


\section{International Journal of Science and Research (IJSR) \\ ISSN (Online): 2319-7064}

Index Copernicus Value (2013): 6.14 | Impact Factor (2014): 5.611

ideally suited to high-volume applications with memory restrictions, or applications where code density is an issue. The key idea behind Thumb is that of a super reduced instruction set. Essentially, the ARM7TDMIS processor has two instruction sets:

The standard 32-bit ARM set. The Thumb set's 16-bit instruction length allows it to approach twice the density of standard ARM code while retaining most of the ARM's performance advantage over a traditional 16-bit processor using 16-bit registers. This is possible because Thumb code operates on the same 32-bit register set as ARM code. Thumb code is able to provide up to $65 \%$ of the code size of $\mathrm{ARM}$, and $160 \%$ of the performance of an equivalent ARM processor connected to a 16-bit memory system.

\section{ii) $\mathrm{CAN}$}

Controller Area Network (CAN) Protocol was initially created by German automotive system developer Robert Bosch in the mid of-1980s for automotive applications as a method for enabling robust serial communication. The aim was to make automobiles more reliable, robust and safe while decreasing wiring tension, weight and complexity. Since its acceptance, the CAN protocol has gained worldwide popularity in industrial automation and automotive/truck applications.

The goal of this application note is to explain some of the basics of CAN and show the benefits of choosing CAN for embedded systems networked applications.

Controller Area Network (CAN) is a serial communication standard that supports distributed real-time control with a very high level of security. CAN provide multi-master capabilities, and is particularly suited to networking "intelligent" devices as well as sensors and actuators within a system or sub-system. CAN bus devices are compatible with nearly every PCI board. However, PCI boards are not compatible with notebook computers.

We are using transceiver MCP2551 and controller MCP2510.

\section{iii) Temperature sensor}

The LM35 series are precision integrated-circuit temperature sensors, whose output voltage is linearly proportional to the Celsius (Centigrade) temperature. The LM35 thus has an advantage over linear temperature sensors calibrated in ${ }^{\circ}$ Kelvin, as the user is not required to subtract a large constant voltage from its output to obtain convenient Centigrade scaling [8]. The LM35 does not require any external calibration or trimming to provide typical accuracies of $\pm 1 / 4^{\circ} \mathrm{C}$ at room temperature and $\pm 3 / 4^{\circ} \mathrm{C}$ over a full -55 to $+150^{\circ} \mathrm{C}$ range.

As it draws only $60 \mu \mathrm{A}$ from its supply, it has very low selfheating, less than $0.1{ }^{\circ} \mathrm{C}$ in still air. The LM35 is rated to operate over a $-55^{\circ}$ to $+150^{\circ} \mathrm{C}$ temperature range, while the $\mathrm{LM} 35 \mathrm{C}$ is rated for $\mathrm{a}-40^{\circ}$ to $+110^{\circ} \mathrm{C}$ range.

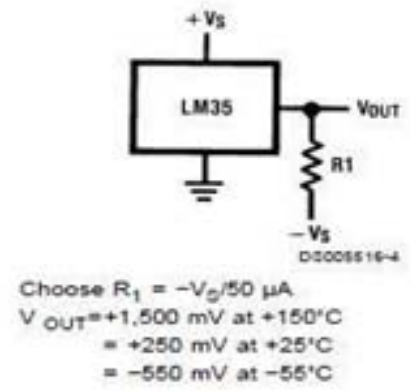

Figure 3: Circuit Diagram of Temperature Sensor

\section{iv) LDR}

LDRs or Light Dependent Resistors are very useful especially in light/dark sensor circuits. Normally the resistance of an LDR is very high, sometimes as high as $1,000,000$ ohms, but when they are illuminated with light, the resistance drops dramatically. Thus in this project, LDR plays an important role in switching on the lights based on the intensity of light i.e., if the intensity of light is more (during daytime) the lights will be in off condition. And if the intensity of light is less (during nights), the lights will be switched on.
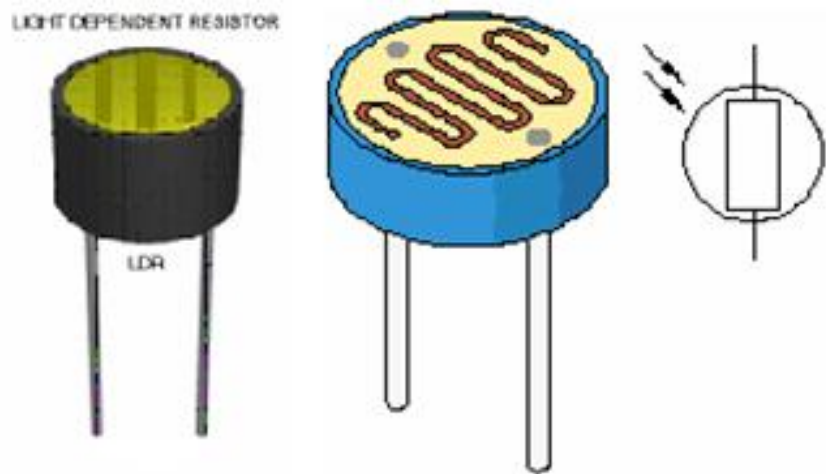

Figure 4: Circuit Diagram of LDR Sensor

\section{v) Reed- switch}

The reed switch is an electrical switch operated by an applied magnetic field. The basic reed switch consists of two identical flattened ferromagnetic reeds, sealed in a dry inertgas atmosphere within a glass capsule, thereby protecting the contact from contamination. The reeds are sealed in the capsule in such a way that their free ends overlap and are separated by a small air gap.

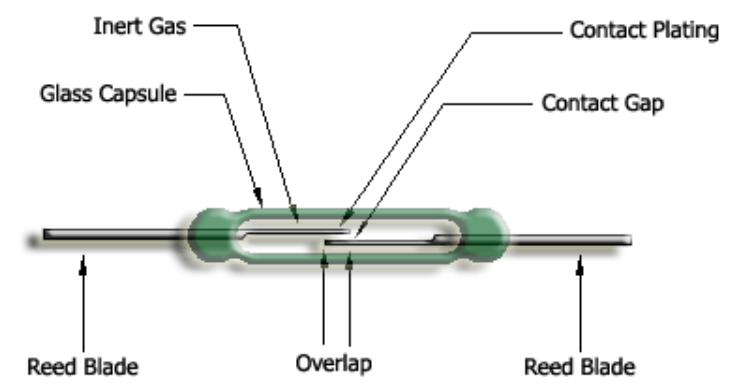

Figure 5: Circuit Diagram of Reed Switch

\section{vi) Buzzers}

These buzzers feature low power consumption, a safe, sparkfree, non-contact structure, a small size and light weight for an easy mounting to printed circuit boards. As a result, an increasing number of piezo ceramic buzzers are now used to 


\section{International Journal of Science and Research (IJSR) \\ ISSN (Online): 2319-7064 \\ Index Copernicus Value (2013): 6.14 | Impact Factor (2014): 5.611}

generate an artificial voice in combination with voice synthesizing ICs. To produce high quality piezo ceramic buzzers, FDK has capitalized on many years of piezo ceramics production and outstanding ceramic processing technologies and thin film forming techniques. By adding a sophisticated audio know-how to this manufacturing expertise, FDK offers a large array of electronic tone generating products, such as piezo ceramic diaphragms, sounders and buzzers, to meet loud sound outputs, wide frequency ranges and many other requirements.

\section{vii) Power Supply}

The input to the circuit is applied from the regulated Power Supply. The ac. input i.e., $230 \mathrm{~V}$ from the solar panel which is absorbed and stored in battery $12 \mathrm{~V}$ and is fed to a rectifier. The output obtained from the rectifier is a pulsating dc voltage. So in order to get a pure dc voltage, the output voltage from the rectifier is fed to a filter to remove any ac components present even after rectification. Now, this voltage is given to a voltage regulator to obtain a pure constant dc voltage.

\section{viii) Software Design}

This includes the coding of ARM7 processor using Embedded c using keil software verson4 and flash magic version9 for dumping.

\section{ix) Advantages of proposed system}

Provides high level safety for industries. Easy retrieval of data for the cause of incidents, low cost, reliable because of its error checking mechanism and less complex system for installing and application.

\section{x) Conclusion}

From the above project we came to conclude that in all the industries there are lot of issues we can rectify the error with some sensors and ARM processor. ARM has lot of advantages and even work for all types of industries with less cost and more accuracy.

\section{xi) Future Enhancement}

There is always chance to improve any system as research \& development is an endless process. This can be further implemented in all small industries with less cost and even in android mobiles to get direct message.

\section{References}

[1] An implementation of a proposed modification of CAN protocol on CAN field bus controller component for supporting a dynamic priority policy. Industry Applications Conference, 2003. 38th IAS Annual Meeting. 12-16 Oct. 2003 Hasnaoui, S. Lab. SYSCOM/ENIT, Ecole Nationale d'Ingenieurs de Tunis, Tunisia Kallel, O. Kbaier, R. Ahmed, S.B. Volume: 1 Page(s): 23 - 31J. Clerk Maxwell, A Treatise on Electricity and Magnetism, 3rd ed., vol. 2. Oxford: Clarendon, 1892, pp.68-73.

[2] Industrial Automation using the CAN Bus Platform White Paper May 2, 2003.

[3] Network protocols used in automotive industry Jon Bell1 July 24, 2002.

[4] Robert Bosch GmbH. CAN specification version 2.0, 1991. Bosch CAN.
[5] Standard-11898, "Road vehicles-interchange of digital Information Controller Area Network (CAN) for highspeed communication," International Standards Organization (ISO), 1993.

[6] Reducing message-length variations in resourceconstrained embedded systems implemented using the Controller Area Network (CAN) protocol Authors: Mouaaz Nahas Embedded Systems Laboratory, University of Leicester, University Road, Leicester LE1 7RH, UK Michael J. PontEmbedded Systems Laboratory, University of Leicester, University Road, Leicester LE1 7RH, UKMichael ShortEmbedded Systems Laboratory, University of Leicester, University Road, Leicester LE1 7RH, UKPublished in: Journal of Systems Architecture: the EUROMICRO Journal archive Volume 55 Issue 5-6, May, 2009 Pages 344-354.

[7] Timely Use of the CAN Protocol in Critical Hard RealTime Systems with Faults Authors: Ian Broster Alan Burns Published in: · Proceeding ECRTS '01 Proceedings of the 13th Euromicro Conference on RealTime Systems Page 95

\section{Author Profile}

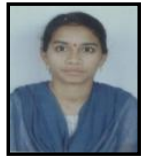

B. Meena Kumari is P.G. Student, Research on Industrial Automation and Process Control System Using Can Bus Department of Electronics and communication Engineering, Satya Institute of Pradesh, India Technology and Management, Vizianagaram, Andhra

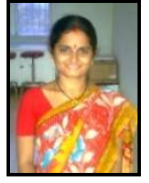

M. Satya Sailaja is Associate Professor, Research Industrial Automation and Process Control System Using Can Bus Department of Electronics and communication Engineering, Satya Institute of Technology and Management, Vizianagaram, Andhra Pradesh, India 